\title{
Study of surface-enhanced infrared absorption spectroscopy (SEIRAS) of black inkjet prints using silver colloid
}

\author{
Michal Oravec $^{\mathrm{a}}$, Katsuya Miurab ${ }^{\mathrm{b}}$ Michal Čeppan ${ }^{\mathrm{a}}$ \\ ${ }^{a}$ Faculty of Chemical and Food Technology, Slovak University of Technology in Bratislava, \\ Radlinského 9, SK-812 37 Bratislava, Slovak Republic \\ ${ }^{b}$ Iwate University, 4-3-5 Ueda, Morioka, Iwate, Japan 020-855 \\ michal.oravec@stuba.sk
}

\begin{abstract}
Only black inkjet prints were studied with regard to forensic examination of documents. We analysed twenty-one inkjet prints using surface-enhanced infrared absorption spectroscopy (SEIRAS). The present paper deals with micro-destructive document analysis based on silver nanoparticles (AgNPs) use to obtain surface enhanced ATR-FTIR (attenuated total reflection - Fourier transform infrared) spectra directly from the document.

For this experiment, AgNPs in colloid form was applied. AgNPs for SEIRAS analyses were synthesised and afterwards analysed by scanning electron microscopy (SEM).

The purpose of the enhancement of colloids was to improve the resolution of the absorption signal of the analysed samples from noise. In the experiment, the obtained ink ATR-FTIR spectra, with and without silver treatment, were significantly affected by the paper base.
\end{abstract}

Keywords: SEIRAS, SEM, inkjet prints, silver colloid

\section{Introduction}

When a spherical metallic nanoparticle (much smaller than the incident radiation wavelength of the source) is irradiated by a plane monochromatic wave, the oscillating electric field causes the conduction electrons to coherent oscillation. Oscillations of conduction electrons are termed particle plasmons, surface plasmons, Mie plasmons, within the dipole approximation of the Mie theory and dipole particle plasmon resonance (DPPR) (Kelly et al., 2003).

Metallic nanoparticles can absorb radiation directly, whereas smooth metal surfaces cannot. Surface plasmons can be excited in small metal particles or surface protrusions but not directly excited on flat metal surfaces (specific geometry is required). These facts are of central importance in the SEIRAS theory explanation (Aroca R., 2006).

SEIRA was first observed by Hartstein et al. (1980), who found that the absorption signal of organic substances thin films on a silicon substrate can be significantly enhanced by Ag evaporation or the Au method. Surface plasmon resonance was found to result in SEIRAS even if it is not fully understood. Plasmon resonance frequency of metallic nanoparticles can be tuned in the infrared domain using their morphologies (Lal et al., 2007).

Since most infrared signatures of organic species and biomolecules are included in the mid-infrared domain, SEIRAS can be used to improve the infrared detection limits of molecules (Verger et al., 2012).
The present work studies the applicability of microdestructive document analysis based on AgNPs to obtain surface enhanced ATR-FTIR (SEIRA) spectra directly from the document. The novelty of this approach is in the measurement of the SEIRA signal of inkjet ink sample directly on the paper surface. This method is relatively fast with minimum destructive effect on the examined evidence.

\section{Experimental}

\section{Sample spectra measurements}

ATR-FTIR measurements were realised using a Nexus 670 FTIR device, in the ATR mode with Smart Dura Scope accessory - diamond crystal, resolution of $4 \mathrm{~cm}^{-1}, 32$ points of scans in the range of 4000 to $700 \mathrm{~cm}^{-1}$. The background was set on aluminium foil before each measurement; this foil has good reflectivity for the infrared radiation, it is hard to become dirty, it is cheap, available and it adheres easily, and the sample has to adhere to the diamond crystal in the Dura Scope.

All samples were measured and compared in the black area spots and black areas with a silver drop. Raw spectra were treated using baseline correction and normalised by $0-1$ normalisation.

\section{Preparation and characterisation of AgNPs colloid}

Silver colloid was prepared according to P. C. Lee and D. Meisel (Lee et al., 1982). The chemical substances for this experiment were extra pure: sodium citrate 
$\left(\mathrm{C}_{6} \mathrm{H}_{7} \mathrm{NaO}_{7}\right.$, pharmaceutical secondary standard, Sigma-Aldrich), silver nitrate $\left(\mathrm{AgNO}_{3} 99.9999 \%\right.$, Sigma-Aldrich). Deionised - freshly boiled water was used for the preparation of all aqueous solutions.

Approximately $2 \mathrm{ml}$ of the silver colloid were poured in a PCR tube and concentrated by centrifugation at $6000 \mathrm{rpm}$ for $15 \mathrm{~min}$ followed by supernatant. The amount of $0.1 \mu \mathrm{l}$ of the remaining centrifuged silver colloid was applied to every sample.

To characterise their shape, size and aggregation, the prepared silver particles (SP's) were checked using a scanning electron microscope (SEM JEOL $7500 \mathrm{~F}$ ) adjusted with the accelerating voltage of $15 \mathrm{kV}$ and magnification in the range of 50000 to 500000 times.

\section{Sampling}

In this paper, twenty-one samples (Tab. 1) of the inkjet-printed documents were examined. Each sample consists of office paper (Xerox Performer $80 \mathrm{~g} / \mathrm{m}^{2}$ ) on which full square areas were printed by different types of office print devices in normal quality. All devices were set to print black ink only. Before the measurement, the ink samples were treated with nanostructured metallic colloid. Fresh synthesised silver colloid from centrifugation was used.

Tab. 1. Inkjet printer devices used for samples preparation.

\begin{tabular}{|c|c|c|}
\hline Sample & Manufacturer & Type of Print Devices \\
\hline $\mathrm{C} 1$ & Canon & IP 3000 \\
\hline $\mathrm{C} 2$ & Canon & Pro 9500 II \\
\hline C3 & Canon & IP 4300 \\
\hline $\mathrm{C} 4$ & Canon & IP 5200 \\
\hline C5 & Canon & IP 7200 \\
\hline $\mathrm{E} 1$ & EPSON & SX 425 \\
\hline E2 & EPSON & PMD 800 \\
\hline E3 & EPSON & PM 830c \\
\hline $\mathrm{E} 4$ & EPSON & PX 730WD - origin. ink \\
\hline E5 & EPSON & DX 7400 \\
\hline E6 & EPSON & PX 730 WD - unoriginal ink \\
\hline E7 & EPSON & P50 - unoriginal ink \\
\hline E8 & EPSON & P50 - origin. Ink \\
\hline E9 & EPSON & SX 130 - unoriginal ink \\
\hline $\mathrm{E} 10$ & EPSON & L 210 \\
\hline $\mathrm{H} 1$ & $\mathrm{HP}$ & Photosmart C4580 \\
\hline $\mathrm{H} 2$ & HP & Photosmart 3210 \\
\hline $\mathrm{H} 4$ & HP & Photosmart C3180 \\
\hline H5 & HP & DeskJet 920C \\
\hline H6 & HP & Photosmart C1410 \\
\hline $\mathrm{H} 7$ & $\mathrm{HP}$ & Office jet 8100 \\
\hline
\end{tabular}

\section{Results and discussion}

The analysis was performed by measuring spectra in the infrared range to obtain signal of the inkjet ink on office paper surface. Twenty-one samples of tree printer manufacturers; Canon, Epson, HewlettPackard (HP) - from our database, were identified. Information about chemical compounds of the used inks was collected from available (MSDS) Material Safety Data Sheets (MSDS online).

Synthesised silver colloid was analysed via SEM microscopy. SEM images (Fig. 1) show a 50 000× magnification of AgNPs after 15 min of centrifugation at $6000 \mathrm{rpm}$. On the SEM image (Fig. 1), islets composed of micrometre size aggregates

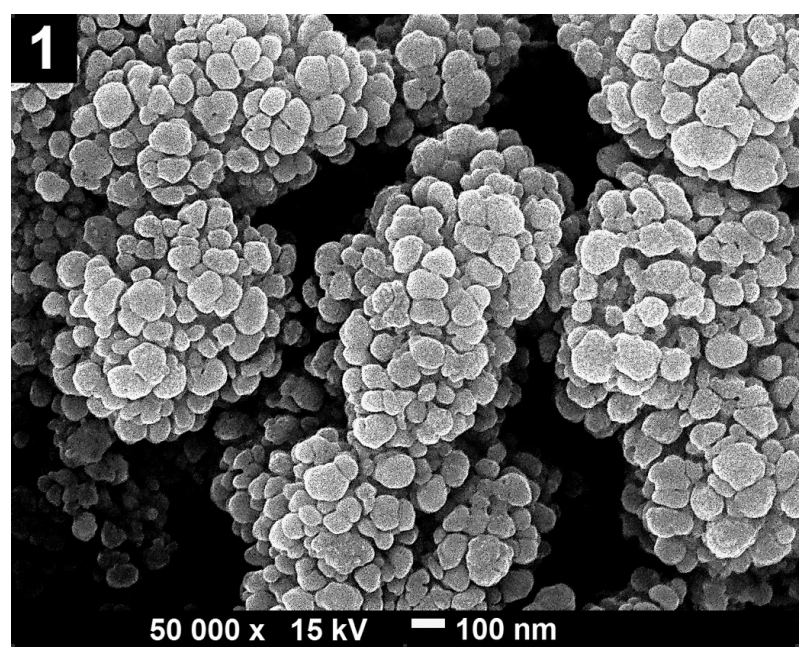

Fig. 1. SEM image of Ag nanoparticles synthesised using sodium citrate as a reducing agent, 50 000×

magnification, scale bar $100 \mathrm{~nm}$. Islet surface composed of aggregates in the size of micrometres.

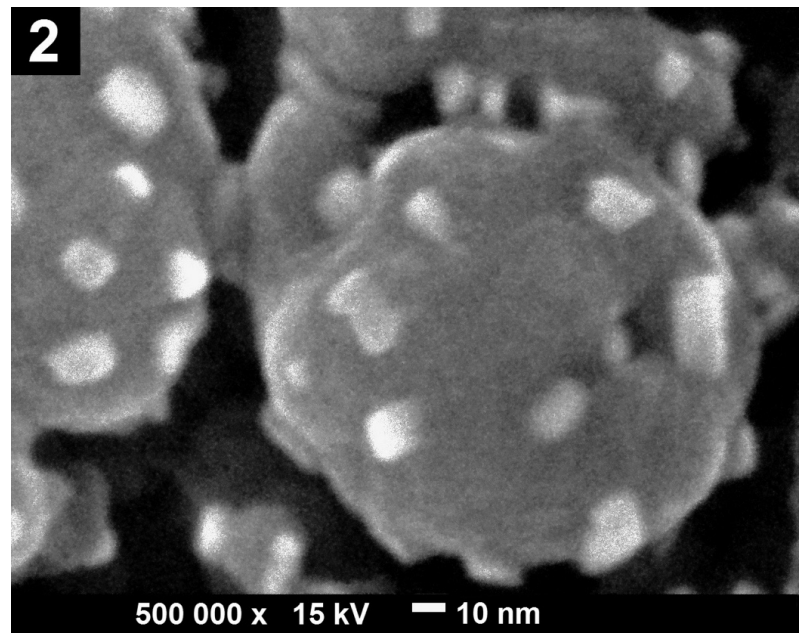

Fig. 2. SEM image of $500000 \times$ magnification of the same AgNPs sample. The particle is spherical in shape with numerous small surface protrusions. 


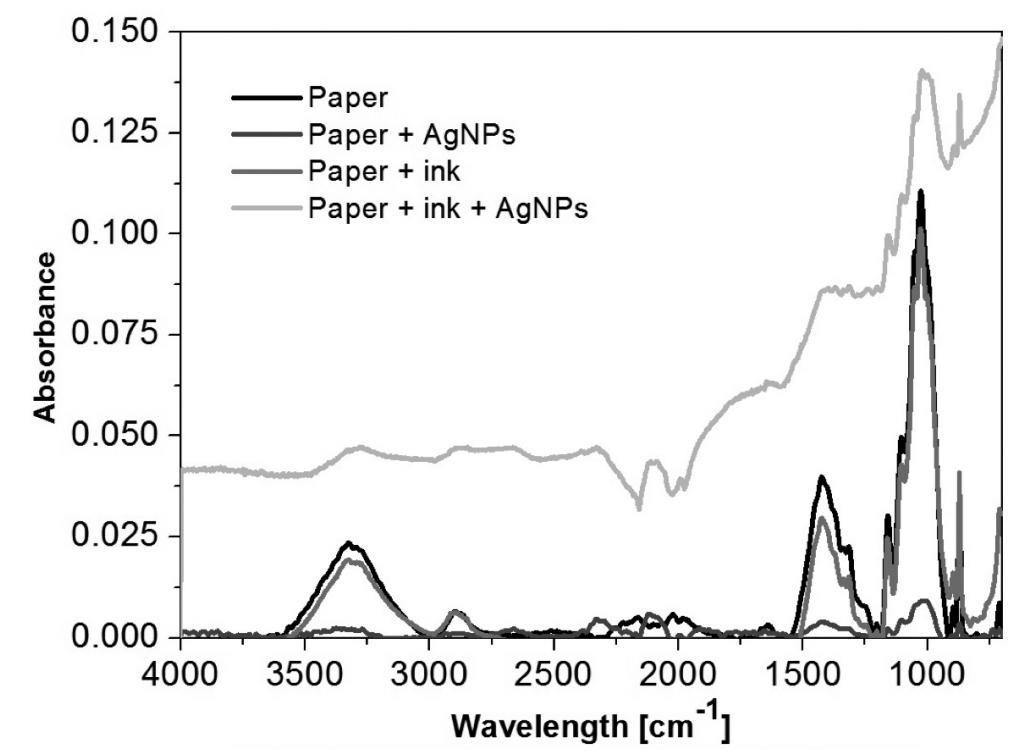

Fig. 3. ATR-FTIR spectra; paper, paper with AgNPs, E10 sample, and E10 with AgNPs.

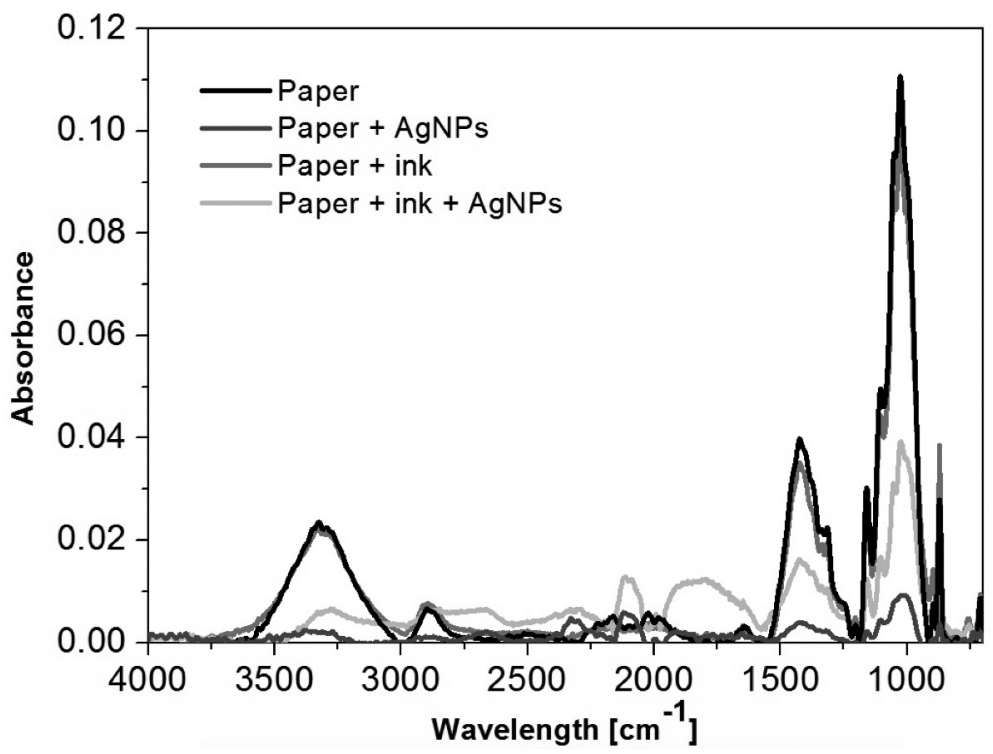

Fig. 4. ATR-FTIR spectra; paper, paper with AgNPs, E10 sample, and E10 with AgNPs.

The spectra were treated using baseline correction.

were observed. The aggregates are built of kidney shaped particles of the size of hundred nanometres approximately. These particles are presented on another SEM image (Fig. 2) showing the $500000 \times$ magnification of the same silver sample. This figure shows that the particles contain surface protrusions of around tens of nanometres and of various multi edge shapes.

The enhancement effect of the prepared silver colloid on the printed office paper samples was studied using an ATR-FTIR device. Thus, obtained spectra are termed SEIRA spectra. The character of untreated office paper spectrum (Fig. 3) is typical of the presence of cellulose in the analysed samples. Intensity of the spectrum of paper with AgNPs is reduced significantly in comparison with untreated paper spectrum. The spectrum of paper with ink belongs to the E10 (Epson $\mathrm{L}$ 210) sample. In general, this spectrum is very similar to that of the untreated paper spectrum, which can be attributed to the significant paper signal overlapping the relatively poor intensity of the ink signal caused by a low amount of the ink printed on the paper.

Spectrum of the E10 sample with AgNPs differs from others due to the increasing trend of the spectral slope. Attenuation of the spectral slope was caused by the application of manual baseline correction. The final ATR-FTIR spectra (Fig. 4) were compared visually. 


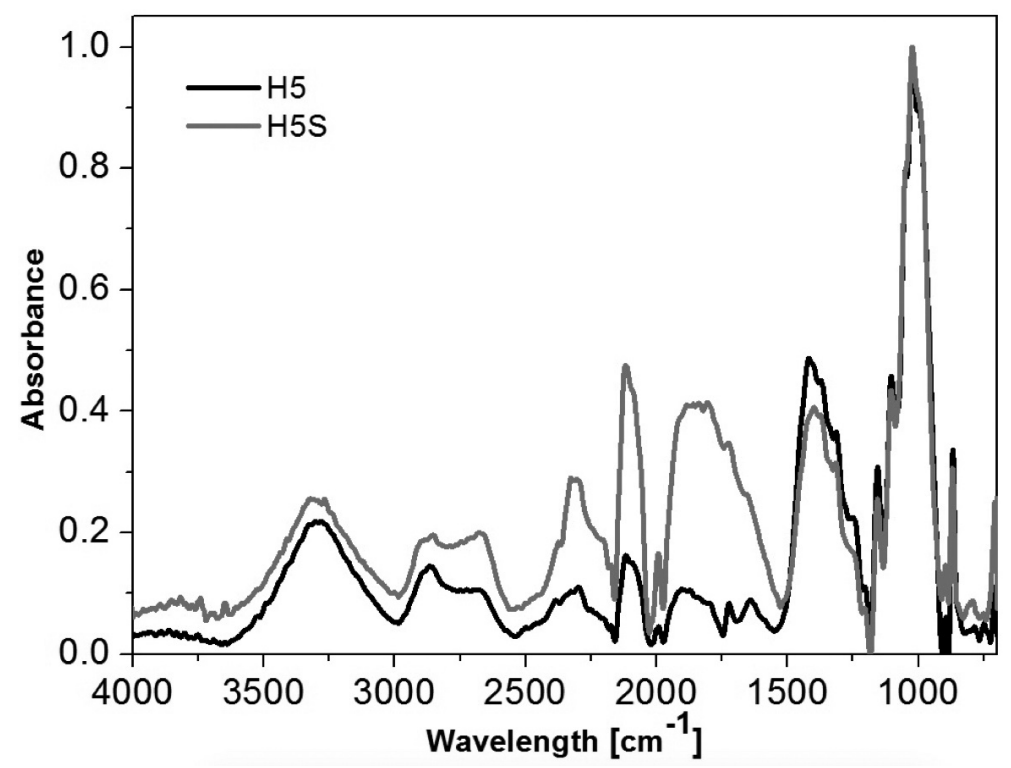

Fig. 5. ATR-FTIR spectra; sample H5 (DeskJet 920C), H5S sample (DeskJet 920C) with AgNPs. The spectra were treated using baseline correction and normalisation procedure.

The best SEIRA spectrum of the analysed database, obtained from sample H5 (Hewlett-Packard, DeskJet 920C), is depicted in Fig. 5.

The most prominent peak in the range of 1950 to $2250 \mathrm{~cm}^{-1}$ (Fig. 5) was attributed to the diamond crystal signal of the Smart Dura Scope accessory of the FTIR device. This band is present in each sample measurement.

In the range from 1500 to $1850 \mathrm{~cm}^{-1}$, the enhancement effect of AgNPs on the ink is evident (Fig. 5). Thus, enhanced band can be attributed to carbonyl groups, and the carbonyl band can be assigned to the presence of 2-pyrrolidone (Fig. 6).

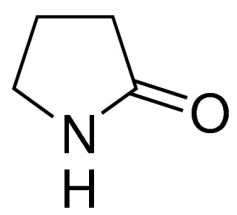

Fig. 6. Chemical structure of 2-pyrrolidone $\left(\mathrm{C}_{4} \mathrm{H}_{7} \mathrm{NO}\right)$ [SigmaAldrich online].

This chemical compound is used in inkjet ink preparation as water-soluble organic solvent/cosolvent (BASF online). According to the available MSDS (MSDS online), each Hewlett-Packard ink contains this chemical compound. As this carbonyl band of 2-pyrrolidone was distinguished in almost each analysed sample (also of non-Hewlet-Packard inks), all samples likely contain this compound.

\section{Conclusions}

Twenty-one inkjet prints were analysed using surface-enhanced infrared absorption spectroscopy (SEIRAS) directly on office paper surface. Synthetised silver colloid was analysed by SEM microscopy.

Intensity of the spectrum of paper with AgNPs is significantly reduced in comparison with that of the untreated paper spectrum; on the other hand, the obtained ink ATR-FTIR spectra with and without silver treatment were significantly affected by the paper base. Enhancement effect of AgNPs is evident in the range from 1500 to $1850 \mathrm{~cm}^{-1}$ attributed to the carbonyl band of 2-pyrrolidone.

\section{Acknowledgement}

The authors would like to thank for financial contribution from the Tatra banka Foundation and the STU Grant scheme for Support of Young Researchers.

\section{References}

Aroca R (2006) Surface-Enhanced Vibrational Spectroscopy, John Wiley \& Sons Inc. ISBN: 978-0-47160731-1.

BASF online. [accessible: 2016-1-26] http://www. standort-ludwigshafen.basf.de/group/corporate/siteludwigshafen/en/brand/2_PYRROLIDONE.

Hartstein A, Kirtley JR, Tsang JC (1980) Physical Review Letters 45: 201-205.

Kelly KL, Coronado E, Zhao LL, Schatz GC (2003) Journal of Physical Chemistry B 107: 668-677. 
Lal S, Link S, Halas NJ (2007), Nature Photonics 1: 641-648.

Lee PC, Meisel D (1982) Adsorption and SurfaceEnhanced Raman of Dyes on Silver and Gold Sols, Journal of Physical Chemistry A 86: 3391-3395.

MSDS online. [accessible: 2017-10-30] http://www.ilpi. $\mathrm{com} / \mathrm{msds}$.
SigmaAldrich online. [accessible: 2017-5-9] http:// www.sigmaaldrich.com/content/dam/sigma-aldrich/ structure0/117/mfcd00005270.eps/_jcr_content/ renditions/mfcd00005270-large.png

Verger F, Pain T, Nazabal V, Boussard-Plédel C, Bureau B et al. (2012) Sensors and Actuators B: Chemical 175: 142-148. 